\title{
Thermal performance difference of ideal gas model and van der Waals gas model in gas-loaded accumulator
}

\section{Shuce Zhang* and Hiromu Iwashita}

Graduate School of Engineering, Yokohama National University, Tokiwadai 79-5 Hodogaya-ku, Yokohama, Kanagama, Japan Email: zhang-shuce-hc@ynu.jp Email: iwashita-hiromu-mw@ynu.jp

*Corresponding author

\author{
Kazushi Sanada \\ Faculty of Engineering, \\ Yokohama National University, Japan \\ Email: sanada-kazushi-sn@ynu.ac.jp
}

\begin{abstract}
Real gas that considering intermolecular force and molecular volume shows difference compared with the assumption as ideal. While designing accumulator or quantitatively evaluating working state of accumulator in hydraulic circuit, charged in gas-loaded accumulator, the gas properties involves pressure, temperature and stored energy are assumed by the first law of thermodynamics and gas equation of state. The discrepancy of thermodynamic behaviours of real gas from ideal gas should be highlighted. In this paper, the well-known van der Waals equation was employed as real gas model to compare with ideal gas model. The gas behaviours have shown different trends during compression and expansion by mathematically analysis and being confirmed in both isothermal and adiabatic operations. A detailed pressure deviation between ideal gas model and van der Waals model was displayed.
\end{abstract}

Keywords: hydraulic; gas-loaded accumulator; thermal properties; equation of state; van der Waals model; gas pressure; isothermal; adiabatic; energy; control.

Reference to this paper should be made as follows: Zhang, S., Iwashita, H. and Sanada, K. (2018) 'Thermal performance difference of ideal gas model and van der Waals gas model in gas-loaded accumulator', Int. J. Hydromechatronics, Vol. 1, No. 3, pp.293-307.

Biographical notes: Shuce Zhang completed his Mechanical Design Bachelor's degree in 2011 at Dalian Minzu University, China, his Mechanical Engineering Master's degree in 2015 from Dalian Polytechnic University, China and now he is a PhD candidate at Yokohama National University, Japan. His research has focused on hydraulic system for hydraulic-hybrid excavator and thermal performance of gas-loaded accumulator. 
Hiromu Iwashita received his Mechanical Engineering Bachelor's degree in March 2018 at Yokohama National University, Japan, now he is a Mechanical Engineering Master student at Yokohama National University. His research focuses on nucleate boiling for water.

Kazushi Sanada received his BS, MS and Doctorate degrees in Control Engineering from the Tokyo Institute of Technology, Japan, in 1984, 1986 and 1996, respectively. He had worked for Tokyo Institute of Technology as a Research Assistant from 1986 to 1998. He had been an Associate Professor in Yokohama National University from 1998 to 2004. Since 2004, he has been a Professor in Faculty of Engineering, Yokohama National University. His research field is modelling and control of fluid power systems including oil-hydraulics and water hydraulics.

\section{Introduction}

The need of rational use of energy is now a necessity that not only for a purely technical nature, but also involves environmental and social issues. Nowadays the hybrid power system has been widely applied to automobiles in Luo et al. (2015) and Meng et al. (2016) and construction machinery successfully (Ishihara and Hoshino, 2017; Kim et al., 2016; Chen and Zhao, 2017). To ease the power demand and supply variation challenge, designs of energy regeneration, energy loss reduction become significant in fluid power system. Hydraulic energy can be stored during periods of high production and low consumption and release energy during periods of low production and high consumption (Li et al., 2011). Technologies including capacitors, thermal storage, battery chemistries, compressed gas energy storage, pumped hydraulic power are used in varies of energy storage systems. More efficient power drive systems are being advanced such as pipeline optimisation and pressure/flow rate pulsation absorption in hydraulic circuits. The gas-loaded accumulator is being used as a key component in hydraulic hybrid power system (Wasbari et al., 2017) which consists of two sides, namely the oil side and the gas side. It stores potential energy through the compression of a dry inert gas (typically nitrogen) in a container open to a relatively incompressible fluid (typically hydraulic oil). There are two types of gas-loaded accumulators commonly used today. One is the bladder type (including diaphragm designs) and the other one is the piston type. While other types of accumulator designs exist, compressed gas accumulators are far and away the most common.

Accumulator can be applied in situations including shock or pulsation dampening, leakage compensation, energy regeneration, thermal expansion and noise reduction. Accumulator operates by making use of the considerable difference in compressibility between a gas and hydraulic fluid. Using the bladder design, the nitrogen in the bladder is highly compressible while the hydraulic fluid in the oil side of the shell is virtually non-compressible. The bladder contained in the shell is pre-charged with nitrogen gas to a pressure calculation determined by system parameters and the work to be done. A piston-type accumulator has a cylindrical chamber in a general way, which has a piston in it. This piston is either spring loaded or some calculated weight is kept on it or even pneumatically pressurised. The hydraulic pump pumps the fluid into the accumulator, which is nothing but a sealed container. The volume of the container is fixed and cannot 
be changed. But the quantity of hydraulic fluid being pumped inside the container is increasing continuously. Then the pressure of the hydraulic fluid inside the container starts to increase.

The gas in the accumulator was assumed to be ideal that making it easy to calculate the gas properties, but this have in many cases also shown to result in large errors as described by Pourmovahed and Otis (1985). In many cases the accumulator in hydraulic system is operated at high pressures at which the fluid properties significantly deviate from those of an ideal gas. As assumption, the ideal gas model takes no account of the intermolecular force and molecular volume that it will perform with different thermodynamical properties compared with real gas. Mohamed and Paraschivoiu (2005) investigated real gas effects at high pressures in a sample problem where hydrogen is released from a high-pressure chamber. Jassim et al. (2008) analysed natural gas behaviour when it is real and how erroneous the properties may become when the gas is assumed to be ideal by analysing the flow of gas through a supersonic nozzle. The volume translation methods for real gas computation has been presented by Matheis et al. (2016).

This paper is focusing on nitrogen gas effects on accumulator operation in hydraulic system. Regarding to modelling accuracy, especially in particular operating conditions such as isotheral or adiabatic in the gas-loaded accumulator, the difference of ideal gas and real gas should be investigated. The van der Waals gas model is employed as the real gas model in this study. The thermodynamical characteristics of two gas models in condition of high-pressured pre-charged gas are analysed. Special attention is devoted to mathematically analyse gas compression and expansion in both isothermal and adiabatic operations. A detailed calculation pressure error between these two models is highlighted. Section 2 is the nomenclature for mathematical expressions in this paper. Section 3 describes accumulator model by energy conservation correlation and gas equation of state in accumulator. Ideal gas model and van der Waals gas model are compared in both charging process and discharging process in Section 4. The thermal difference of ideal gas model and van der Waals gas model is discussed in Section 5 and the main work is concluded in Section 6 .

\section{Nomenclature}

$C_{v}$ specific heat at constant volume

$m$ amount of gas

$m_{i}$ amount of ideal gas

$m_{r}$ amount of real gas

$P_{0} \quad$ initial gas pressure

$P \quad$ gas pressure

$P_{i} \quad$ ideal gas pressure

$P_{r}$ real gas pressure

$Q$ heat energy 
$R$ gas constant

$S$ entropy

$T_{0}$ initial gas temperature

$T$ gas temperature

$T_{c}$ critical temperature

$U$ internal energy

$V_{0}$ initial gas volume

$V \quad$ gas volume

$v_{c} \quad$ critical mole volume

$W$ performed work.

\section{Accumulator models}

\subsection{Thermal energy balance}

A hydraulic accumulator is a pressure storage reservoir in which a non-compressible hydraulic fluid is held under pressure that is applied by an external source. During the gas compression and expansion process in accumulator, gas temperature varies and the heat is transferred between the charged gas and the accumulator wall. To model the thermal energy balance in the accumulator gas, a thermodynamical model is set up using the first law of thermodynamics.

Figure 1 Energy balance of a gas-loaded accumulator (see online version for colours)

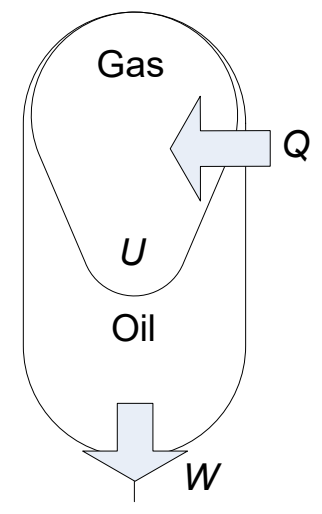

Nitrogen gas has internal energy of $U$ as shown in Figure 1. The nitrogen gas receives heat energy $Q$ from ambient and performs work $U$ to outside. Mathematical modelling begins by the conservation of energy equation:

$$
d U=d Q-d W
$$


Definition of entropy can be described as

$$
d S=\frac{d Q}{T}
$$

and rate of work output is:

$$
d W=P d V
$$

Substituting equation (2) and equation (3) to equation (1) yields:

$$
d U=T d S-P d V
$$

The total differential of internal energy is:

$$
d U=\left(\frac{\partial U}{\partial T}\right)_{V} d T+\left(\frac{\partial U}{\partial V}\right)_{T} d V
$$

The constant volume specific heat $C_{v}$ is defined as

$$
C_{v}=\frac{1}{m}\left(\frac{\partial U}{\partial T}\right)_{V}
$$

To eliminate the internal energy $d U$, its total differential formula is expressed by:

$$
d U=m C_{v} d T+\left(\frac{\partial U}{\partial V}\right)_{T} d V
$$

The differential form of $U$ with respect to volume of above equation is

$$
\left(\frac{\partial U}{\partial V}\right)_{T}=T\left(\frac{\partial S}{\partial V}\right)_{T}-P
$$

From the Maxwell relationship, it is known that

$$
\left(\frac{\partial S}{\partial V}\right)_{T}=\left(\frac{\partial P}{\partial T}\right)_{V}
$$

Then the equation (5) can be written as

$$
\left(\frac{\partial U}{\partial V}\right)_{T}=T\left(\frac{\partial P}{\partial T}\right)_{V}-P
$$

The equation (7) is then expressed by:

$$
d U=, C_{v} d t+T\left(\frac{\partial p}{\partial T}\right)_{V} d V-P d V
$$

The partial differential form of $P$ with respect to $T$ is decided by the gas state in accumulator calculated by gas model, which describes the relation between gas pressure, gas temperature and gas volume. 


\subsection{Gas models}

Gas equation of state properly expresses thermal motion with considering mass of molecule. The accumulator gas is often modelled as ideal gas which fails to account for intermolecular force and molecular volume as expressed below:

$$
P V=m R T
$$

The assumptions are derived from the kinetic theory of gases that the only interaction between molecules is elastic collisions (without intermolecular force). The gas consists of molecules that are in ceaseless random motion and the size of the molecules is small relatives to the distances separating them. However practical experience has shown that the ideal gas model has limited accuracy at low pressures. Real gas properties should be considered and therefore quantify the difference gained by using a real gas approach compared to the ideal gas model.

Figure 2 Real gas concept
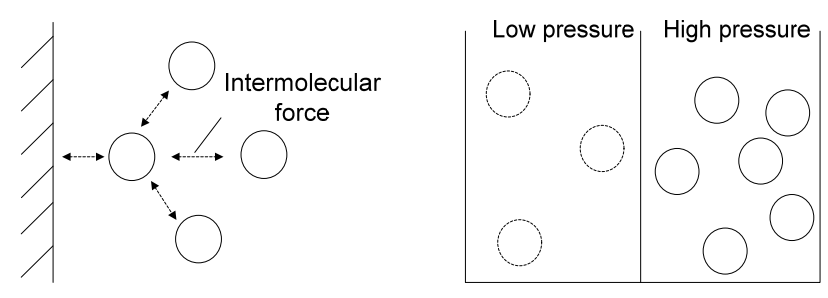

The real gas concept is illustrated in Figure 2 that the intermolecular forces between molecules decrease the gas pressure to the reservoir wall. All the molecules in a sealed volume will have a relatively lower pressure compared with gas model that neglects intermolecular force. In addition, at low pressure the molecule volume drawn by dash line is negligible compared to reservoir volume. Assuming molecules of an ideal gas have zero volume, the volume available to them for motion is always the same as the volume of the container. In contrast, the molecules of a real gas have small but measurable volumes. At low pressures, the gaseous molecules are relatively far apart, but as the pressure of the gas increases, the intermolecular distances become smaller and smaller. As a result, the volume occupied by the molecules becomes significant compared to volume of the reservoir. Consequently, the total volume occupied by the gas is larger than the volume predicted by the ideal gas law (Brown et al., 1994). More accurately, there are equations attempt to consider the 'real' thermodynamical properties such as van der Waals equation:

$$
\begin{aligned}
& \left(P+\frac{a m^{2}}{V^{2}}\right)(V-m b)=m R T \\
& a=\frac{1}{3} v_{c} ; b=\frac{9}{8} R T_{c} v_{c}
\end{aligned}
$$

The constants $a$ and $b$ have positive values and are characteristic of the individual gas. The van der Waals equation of state approaches the ideal gas law shown in equation (12) as the values of these constants approach zero. The constant $a$ provides a correction for the intermolecular forces. Constant $b$ is a correction for finite molecular size and its value 
is the volume of one mole of the atoms or molecules. According to the ideal gas model shown in equation (12) or the van der Waals gas model shown in equation (13), the relation between pressure, volume and temperature is clear to evaluate energy change shown in equation (11). Here the van der Waals gas model is employed as real gas model.

\section{Charging and discharging of accumulator}

\subsection{Compression and expansion}

A gas-loaded accumulator uses a bladder or piston to separate gas and hydraulic fluid. With fluid charging or discharging, the gas is compressed or expanded. Figure 3 shows that pressures of real gas model and ideal gas model corresponding to identical gas volume vary with different trends. From the point $\left(V_{0}, P_{0}\right)$ that real gas pressure is equal with ideal gas pressure, which can be regarded as initial point, to the left side the real gas pressure is higher than ideal gas pressure with decreasing gas volume (as gas charging process), however, to the right side the trend is opposite with increasing gas volume (as gas discharging process).

Figure 3 Pressure relation of ideal gas and real gas models

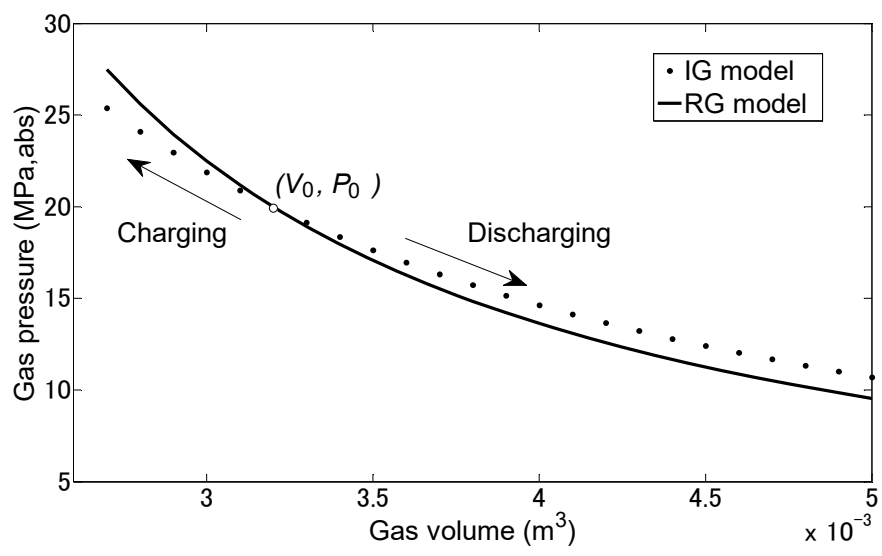

To explain the pressure difference of gas models in charging and discharging processes, assuming comparing these two models that the gas volume and temperature are as same in a single working cycle, the ideal gas pressure can be described by:

$$
P_{i}=\frac{m_{i} R T}{V}
$$

and real gas pressure is:

$$
P_{i}=\frac{m_{i} R T}{V-m_{r} b}-\frac{a m_{r}^{2}}{V^{2}}
$$

The gas pressure in every working cycle and the original gas amount $m_{r}$ and $m_{i}$ are different. Setting the identical initial pressure: 


$$
\mathrm{P}_{r 0}=P_{i 0}
$$

The relationships of $m_{r}$ and $m_{i}$ in these two models can be obtained:

$$
m_{i}=\frac{V_{0} m_{r}}{V_{0}-m_{r} b}-\frac{a m_{r}^{2}}{V_{0} R T_{0}}
$$

As the gas amount is constant in sealed volume, their magnitude relationships will keep constant during accumulator charging and discharging. Combing equation (14), equation (15) and equation (17) yields:

$$
\frac{P_{r}}{P_{i}}=\frac{\frac{V}{V-m_{r} b}-\frac{a m_{r}}{R T V}}{\frac{V_{0}}{V_{0}-m_{r} b}-\frac{a m_{r}}{R T_{0} V_{0}}}
$$

Due to two variables $V$ and $T$ in the right side of this equation, it is difficult to discriminate the value of equation (18). Considering the realistic accumulator operates that the gas compression or expansion happens between two extreme cases isothermal and adiabatic changes, the detailed properties will be described in next subsection. For the isothermal operating:

$$
T=T_{0}
$$

A function of $h(x)$ is created in which the variable $T$ in equation (18) is substituted by constant $T_{0}$ and the $x$ represents the only variable $V$ :

$$
h(x)=\left(\frac{x}{x-m_{r} b}-\frac{a m_{r}}{R T_{0} x}\right) /\left(\frac{V_{0}}{V_{0}-m_{r} b}-\frac{a m_{r}}{R T_{0} V_{0}}\right)
$$

For the adiabatic operating, the $T$ rises during charging and drops during discharging compared with initial temperature $T_{0}$. Give $x$ in $h(x)$ the value $V$ from equation (18), the comparison of $P_{r} / P_{i}$ with $h(V)$ can be predicated by the comparison of $T$ with $T_{0}$. While $T$ equals to $T_{0}, P_{r} / P_{i}$ is equal to $h(V)$ and a higher value of $T$ will lead to a higher value of $P_{r} / P_{i}$, thus higher than $h(V)$. Therefore, the value of equation (18) can be compared with function $h(V)$ based on this relation. Parameter values in equation (20) can be set according to accumulator gas (nitrogen) properties and accumulator operating conditions. The molecular number of real gas $m_{r}$ can be calculated by which in ideal gas model. These parameters are listed in Table 1.

Table 1 Parameters in function $h(x)$

\begin{tabular}{lcc}
\hline Parameter & & Value \\
\hline vdW parameter & $a$ & 0.1062 \\
vdW parameter & $b$ & $2.7009 * 10^{-5}$ \\
Amount of real gas & $m_{r}$ & $40.5142 \mathrm{~mol}$ \\
Gas constant & $R$ & $8.3145 \mathrm{~J} /(\mathrm{mol} \cdot \mathrm{K})$ \\
Initial gas temperature & $T_{0}$ & $303 \mathrm{~K}$ \\
Initial gas volume & $V_{0}$ & $2.7 * 10^{-3} \mathrm{~m}^{3}$ \\
\hline
\end{tabular}


The graph of $h(x)$ is shown in Figure 4. The initial volume is $V_{0}$ in operating process. It indicates that the $h(x)$ is higher than 1.0 for $0<x<V_{0}$. This section can be regarded as accumulator charging process that the gas is contradictorily compressed as the occupied molecule volume is extruded. While $x$ varies between $V_{0}<x<V_{1}$, the value of $h(x)$ is lower than 1.0 which can be regarded as accumulator discharging process that the intermolecular force between gas molecules decreases its pressure to reservoir wall. After a completed discharging process, the low pressure can be set as pre-charged pressure as accumulator has a fixed maximum volume installed in hydraulic system. Thus, the pressure difference of ideal gas and real gas can be neglected. The value of $P_{r} / P_{i}$ can be recognised by the relation depicted in Table 2 . Here according to gas thermodynamic behaviours and accumulator operating performances, mathematically we can explain the higher real gas pressure during gas compression and lower real gas pressure during gas expansion compared with ideal gas model.

Figure 4 Value of function $h(x)$

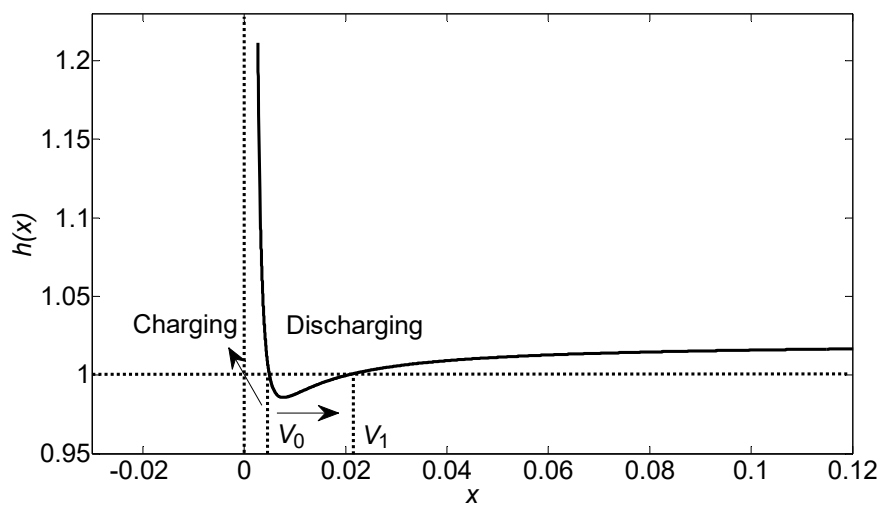

Table 2 Comparison of $P_{r}$ and $P_{i}$ from $h(x)$

\begin{tabular}{|c|c|c|c|}
\hline Charging & $0<V<V_{0}$ & $T>T_{0}$ & $\frac{P_{r}}{P_{i}}>h(V)>1$ \\
\hline Discharging & $V_{0}<V<V_{1}$ & $T<T_{0}$ & $\frac{P_{r}}{P_{i}}<h(V)<1$ \\
\hline
\end{tabular}

\subsection{Isothermal operation and adiabatic operation}

Generally, the accumulator is operated between isothermal process and adiabatic process. During accumulator charging, the hydraulic liquid flows into accumulator, the bladder for a bladder-type accumulator is compressed by hydraulic oil and its volume reduces and relatively the gas pressure increases. The hydraulic energy is transformed into pressurised potential energy stored in accumulator. The energy balance is shown in Figure 1. The gas in accumulator having amount has internal energy of $U$ and receives heat energy $Q$ from the environment and performs work $W$ to the working fluid. During the gas compression and expansion, the gas temperature varies and the heat is transferred between the charged gas and the accumulator wall. Thermodynamic losses occur during these processes as 
described by Pourmovahed and Otis (1990). The gas charging trend in accumulator will affect internal energy and heat transfer. The thermodynamic loss during compression and expansion has been analysed in terms of thermal-time constant and energy storage efficiency by Tanaka and Nakano $(1991,1992)$.

The gas charging and discharging trends are operated between two extreme cases isothermal and adiabatic changes. Isothermal operation maintains a constant temperature which requires the rate of expansion and compression of the gas at such a rate that the gas temperature remains relatively constant. This process is accomplished by slowly expand and/or compress gas. The heat generated in compression process is dissipated through the walls of the accumulator and, in the case of expansion; heat is drawn in through the wall of the accumulator, thus, maintaining a relatively stable gas temperature. Based on equation (19), the gas pressure of van der Waals model can be expressed by:

$$
P=\left(P_{0}+\frac{a m^{2}}{V_{0}^{2}}\right)\left(\frac{V_{0}-m b}{V-m b}\right)-\frac{a m^{2}}{V^{2}}
$$

In adiabatic operation, the gas temperature inside the accumulator increases/decreases at a high rate due to the rapid compression or expansion of the gas. While gas being compressed, the generated heat cannot be dissipated through the wall of accumulator in time, which can be approximately expressed by:

$$
\dot{Q}=0
$$

The energy is transferred only as work, by combining the first law of thermodynamics and gas equation of state by van der Waals model, the gas pressure can be expressed by:

$$
\begin{aligned}
& P=\left(P_{0}+\frac{a m^{2}}{V_{0}^{2}}\right)\left(\frac{V_{0}-m b}{V-m b}\right)^{H}-\frac{a m^{2}}{V^{2}} \\
& H=\frac{R}{c_{v}}+1>1
\end{aligned}
$$

Figure 5 Isothermal and adiabatic compression of gas-loaded accumulator

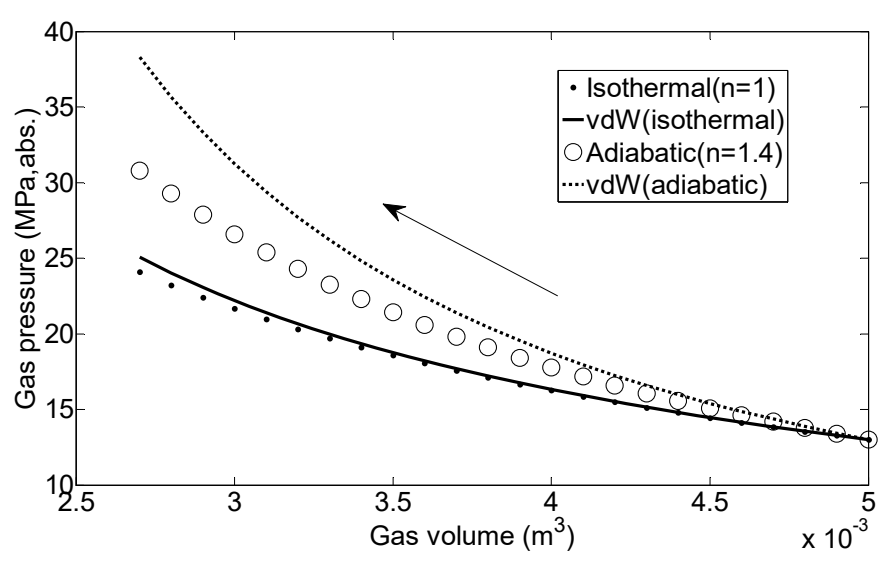


Respectively isotheral and adiabatic operations were calculated by ideal gas model and van der Waals gas model. In ideal gas model, the polytropic index is assumed as 1.0 for isothermal process and assumed as 1.4 for adiabatic process. The initial pressure is assumed as $13 \mathrm{MPa}$, abs for compression and assumed as $33 \mathrm{MPa}$, abs for expansion. Figure 5 shows isothermal and adiabatic compression of gas-loaded accumulator. Gas volume decreases to $2.7 \mathrm{~L}$ from $5 \mathrm{~L}$. The solid line and dot line are isothermal compressions of van der Waals model and ideal gas model, respectively. The dashes line and circle line are adiabatic compressions of van der Waals model and ideal gas model, respectively. The pressure of real gas model is always higher than obtained with ideal gas model. The adiabatic compression leads to more obvious distinction between ideal gas model and van der Waals model. And in the case of expansion process shown in Figure 6, gas volume increases to $5 \mathrm{~L}$ from $2.7 \mathrm{~L}$. The solid line and dot line are isothermal expansions of van der Waals model and ideal gas model, respectively. The dashes line and circle line are adiabatic expansions of van der Waals model and ideal gas model, respectively. The pressure of van der Waals gas model is always lower than obtained with ideal gas model. The adiabatic compression leads to more obvious distinction between ideal gas model and van der Waals model with respect to isothermal compression as well. Both isothermal and adiabatic operations are in accord with previous mathematical analysis.

Figure 6 Isothermal and adiabatic expansion of gas-loaded accumulator

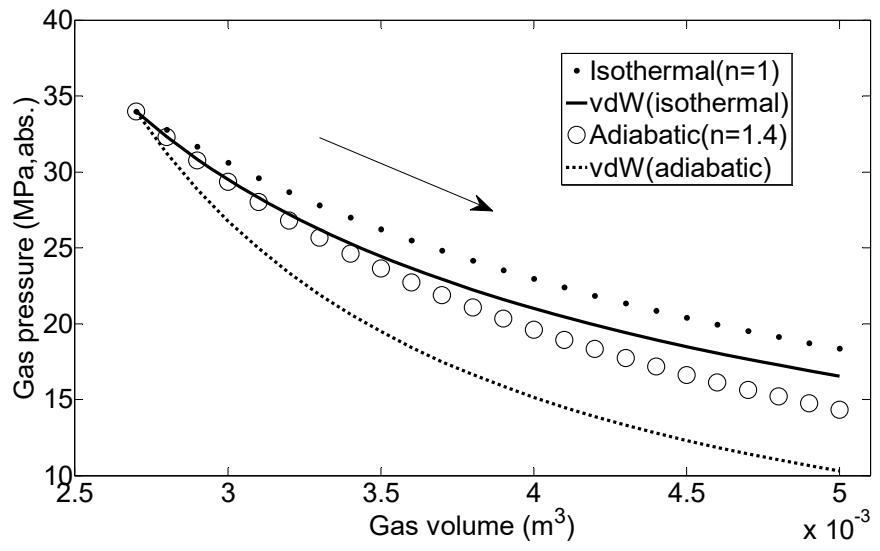

\section{Difference of ideal gas model and van der Waals gas model}

Proper design of a high-pressure accumulator can be achieved by real gas model which is more accurate to evaluate the actual gas behaviours. At low-pressure level operation, the molecular volume is negligible compared with the total gas volume. The ideal gas model provides a good approximation of real gas. Thus, the difference between real gas model and ideal gas model is negligible in low-level pressure. However, the difference should be quantitatively evaluated while the accumulator works from low-pressure range to high-pressure range. It can be concluded that with pressure increasing, the errors of ideal gas model from actual gas behaviour will increase as well. The various applications of 
gas-loaded accumulator (such as energy storage (Hadjipaschalis et al., 2009), shock damper (Wang and Quan, 2014), pulsation absorber (Xiaohui et al., 2015) and energy regeneration (Yagisawa et al., 2016)) puts forward different demands of accumulator such as charging and discharging time, gas volume compression ratio and hydraulic system pressure range et al. These will consequently have a substantial impact on gas thermodynamic properties, therefore the difference of ideal gas model and real one should be highlighted.

Figure 7 Comparison of real gas model and ideal gas model at high pressure and low pressure

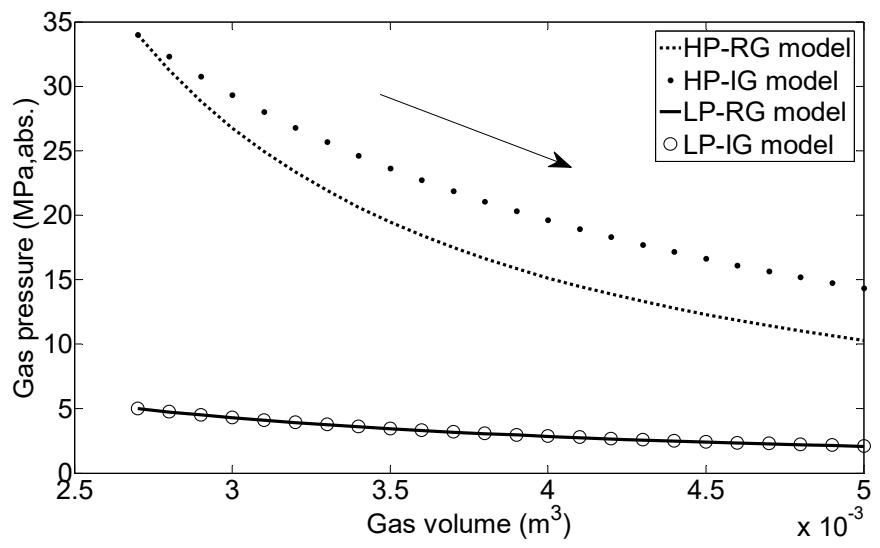

Comparing the gas expansion process respectively from high and low initial pressure, it is seen from Figure 7 that high-pressure real gas model (HP-RG model) shows significant deviation from the pressure behaviour expected for high-pressure ideal gas model (HP-IG model). The pressure deviation can be $3 \sim 5 \mathrm{MPa}$, abs while given a $34 \mathrm{MPa}$, abs initial pressure. Only at relatively low pressure does the pressure variation trend by real gas model (LP-RG model) approximate that by ideal gas model (LP-IG model).

To figure out how the error rate varieties coupled with increasing pressure and gas volume compression ratio, a variety of models are compared by starting from different initial pressure and different gas volume compression ratio and yet same initial temperature. While accumulator is charging, the gas is compressed and a volume decrease will be produced. The compression ratio is described as:

$$
C R=\frac{V_{0}}{V}
$$

The graphical representation of these functions is shown in Figure 8. The horizontal axis is initial gas pressure and the vertical axis represents a ratio of the pressure deviation of ideal gas model and van der Waals gas model with respective to the initial pressure. The pressure deviation is evaluated by calculating the difference between the pressure of the ideal gas model and that of the van der Waals model. The increasing initial pressure will lead to an increase of pressure error ratio of ideal gas from actual gas behaviour. Applications of accumulator, which means working in specific conditions, have different acceptability of pressure error in hydraulic system designing. The gas volume compression ratio plays an important role as well. With a larger volume variation, the pressure error will be higher at a small pressure variation range. For the case of $C R=1.1$ 
shown in Figure 8, the pressure error will reach $10 \%$ at $35 \mathrm{MPa}$, abs which means an error of $3 \mathrm{MPa}$, abs will be produced in high-pressured accumulator. The variation in volume that can be achieve in practice is less than $30 \%$ compared to that achievable by ideal gas model. The pressure error is large enough to lead to difference of accumulator sizing for a hydraulic system. This might result in a damage since the maximum pressure achievable by a commercial accumulator may not exceed about $35 \mathrm{MPa}$, abs.

Figure 8 Relation of pressure error ratio with initial pressure in series of different volume compression ratios

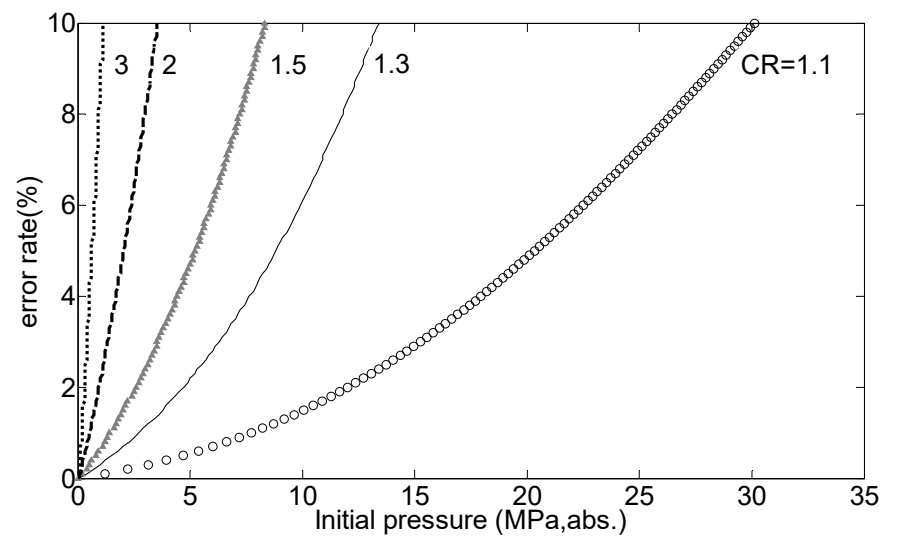

Accumulator application purpose and working pressure decide how the gas model impacts hydraulic system performance. For instance, an accumulator group using in a liquid-piston power generation system for energy storage operates in nearly isothermal process with a $2.1 \sim 10 \mathrm{MPa}$, abs pressure range, the pressure deviation by different gas models can be neglected. In some heavy machines, especially running machine with a hydraulic accumulator, the working pressure can approach 20 32 $\mathrm{MPa}$, abs and the limited space for hydraulic components requires an accurately sizing design of accumulator, the pressure deviation should be considered accordingly.

\section{Conclusions}

As a gas-loaded accumulator enables a hydraulic system to cope with extremes of demand to respond quickly to a temporary demand, the kinetic energy recoverability should be evaluated considering the real gas thermodynamical behaviours and realistic operating conditions. This paper provides an advanced theoretical analysis for sizing accumulator using in varies of hydraulic systems. Thermodynamic behaviours of gas-loaded accumulator by ideal gas model and van der Waals gas model are compared. The authors mathematically analysed the gas pressure variation trends during accumulator charging and discharging processes. To confirm the analysis result, accumulator isothermal and adiabatic operations are simulated, putting particularly in evidence the aspects, relevant from a high-pressure point of view.

The comparison performed shows that, in the van der Waals gas model which taking both intermolecular force and molecular volume into account, the molecular volume 
produces more significant difference from ideal gas model. Results revealed that gas compressibility significantly impacts the volumetric behaviour of gas and thus the size of high-pressure accumulators. Strong differences appear between the results obtained by using ideal gas model and van der Waals model, especially the adiabatic compression operation. The quantitative evaluation of pressure error has been presented. Thermal properties of gas can be determined with the ideal gas model assumption as long as the operating conditions remain in the area of low-pressure range (less than $10 \mathrm{MPa}$, abs). More than $10 \%$ pressure deviation was produced giving a $20 \sim 32 \mathrm{MPa}$, abs working pressure, however, the maximum pressure achievable by a commercial accumulator may not exceed about $35 \mathrm{MPa}$, abs. The variation in volume that can be achieve in practice is less than $30 \%$ compared to that achievable by ideal gas model. For higher compression ratio in accumulator operating with a larger volume variation, the pressure error will reach high values at a small pressure variation range. As of now the ideal gas model coupled with regulation factor based on experiment data is being employed by many accumulator manufacturers or hydraulic system design engineers. The highlighted pressure deviation by gas models and operating conditions should be considered for accumulator design.

\section{References}

Brown, T.L. et al. (1994) Chemistry: The Central Science, Prentice Hall, the University of Michigan.

Chen, M. and Zhao, D. (2017) 'The gravitational potential energy regeneration system with closed-circuit of boom of hydraulic excavator', Mechanical Systems and Signal Processing, Vol. 82, pp.178-192.

Hadjipaschalis, I., Poullikkas, A. and Efthimiou, V. (2009) 'Overview of current and future energy storage technologies for electric power applications', Renewable and Sustainable Energy Reviews, Vol. 13, No. 6, pp.1513-1522.

Ishihara, S. and Hoshino M. (2017) Construction Machine, US Pat. No. 9739037, Washington, DC.

Jassim, E., Abdi, M.A. and Muzychka Y. (2008) 'Computational fluid dynamics study for flow of natural gas through high-pressure supersonic nozzles: part 1. Real gas effects and shockwave’, Petroleum Science and Technology, Vol. 26, No. 15, pp.1757-1772.

Kim, H., Yoo, S., Cho, S. et al. (2016) 'Hybrid control algorithm for fuel consumption of a compound hybrid excavator', Automation in Construction, Vol. 68, pp.1-10.

Li, P.Y. et al. (2011) 'Compressed air energy storage for offshore wind turbines', International Fluid Power Exposition, Las Vegas, NV.

Luo, Y., Chen, T., Li, K. (2015) 'Multi-objective decoupling algorithm for active distance control of intelligent hybrid electric vehicle', Mechanical Systems and Signal Processing, Vol. 64, pp. $29-45$.

Matheis, J., Muller, H., Lenz, C. et al. (2016) 'Volume translation methods for real-gas computational fluid dynamics simulations', The Journal of Supercritical Fluids, Vol. 107, pp.422-432.

Meng, F., Shi, P., Karimi, H.R. et al. (2016) 'Optimal design of an electro-hydraulic valve for heavy-duty vehicle clutch actuator with certain constraints', Mechanical Systems and Signal Processing, Vol. 68, pp.491-503.

Mohamed, K. and Paraschivoiu, M. (2005) 'Real gas simulation of hydrogen release from a high-pressure chamber', International Journal of Hydrogen Energy, Vol. 30, No. 8, pp.903-912. 
Pourmovahed, A. and Otis, D.R. (1985) 'An algorithm for computing nonflow gas processes in gas springs and hydropneumatic accumulators', Journal of Dynamic Systems, Measurement and Control, Vol. 107, No. 1, pp.93-96.

Pourmovahed, A. and Otis, D.R. (1990) 'An experimental thermal time-constant correlation for hydraulic accumulators', Journal of Dynamic Systems, Measurement and Control, Vol. 112, No. 1, pp.116-121.

Tanaka, Y. and Nakano, K. (1991) 'Energy balance of bladder type hydraulic accumulator (first report: experimental investigation of thermal time constant)', Journal of the Japan Hydraulics and Pneumatics Society (in Japanese), Vol. 22, No. 6, pp.666-672.

Tanaka, Y. and Nakano, K. (1992) 'Energy balance of bladder type hydraulic accumulator (second report: estimation of efficiency for energy storage during continuous process)', Journal of the Japan Hydraulics and Pneumatics Society (in Japanese), Vol. 23, No. 3, pp.317-323.

Wang, C. and Quan, L. (2014) 'Methods of restrain the hydraulic impact with active adjusting the variable damping in system with large inertia load', Journal of Mechanical Engineering (in Chinese), Vol. 50, No. 8, pp.182-188.

Wasbari, F., Bakar, R.A., Gan, L.M. et al. (2017) 'Pre-charge pressure effects on isothermal and adiabatic energy storage capacity for dual hybrid hydro-pneumatic passenger car driveline', Proceedings of Mechanical Engineering Research 2017, pp.3-4.

Xiaohui, L., Shuping, C., Weijie, S. et al. (2015) 'Analysis and design of a water pump with accumulators absorbing pressure pulsation in high-velocity water-jet propulsion system', Journal of Marine Science and Technology, Vol. 20, No. 3, pp.551-558.

Yagisawa, R., Ito, K. et al. (2016) 'Control and energy performance improvement with boosting suction line for excavators', in The Proceedings on Autumn Conference of Japan Fluid Power System Society 2016 (in Japanese), Tokyo, Japan, pp.78-80. 